\section{Transnasal Pharyngoesophagogastroduodenoscopy (T-PEGD) in a Patient with Acute Upper Gastrointestinal Bleeding}

Upper gastrointestinal endoscopy is the investigation of choice in patients presenting with acute upper gastrointestinal bleeding. We report here on the use of the transnasal route for endoscopy in a patient whose jaws were wired after he had undergone surgery for a fractured mandible.

A 44-year-old man presented with a 24-hour history of melena and clinically showed orthostatic changes in pulse and blood pressure. Three months earlier, he sustained a right mandible fracture during a brawl with his friend. Subsequently, he underwent three surgical repairs, the most recent one done ten days previously, involving bone graft placement. As the patient's mouth was wired shut, it was elected to carry out endoscopy transnasally, after anesthetizing the right nares with lidocaine jelly. An Olympus GIF-N30 fiberoptic endoscope was used $(5.3 \mathrm{~mm}$ outer diameter, $925 \mathrm{~mm}$ working length). On endoscopic examination, the nasopharynx and pharynx did not show any associated traumatic lesion. At the lower esophagus, a $3.0 \times 1.5 \mathrm{~cm}$ ulcer was seen, with a blood clot and a visible vessel. Using a sclerotherapy needle (Microvasive, Variject 1123, 23 gauge), a total of $2 \mathrm{ml}$ of 1:10000 epinephrine was injected around the vessel. The rest of the evaluation, as far as the descending duodenum, was normal. During the remaining hostpital stay, the patient had no further bleeding. On follow-up at two months, his hemoglobin and hematocrit were stable.

Conventional upper gastrointestinal endoscopy for acute gastrointestinal bleeding may not be possible in patients with lesions of the oral cavity, jaws, and temporomandibular joints that restrict oral intubation. In this case report, we have demonstrated the feasibility of using the transnasal route. Leisser et al. (1) successfully passed an Olympus XQ-10 scope through the nose, and identified a duodenal ulcer with stigmata of recent bleeding. Johnson et al. carried out esophagogastroscopy via the transnasal route in a patient with trismus (2). The transnasal endoscopy technique has also been used to place feeding tubes (3). Using twenty healthy volunteers, Shaker (4) demonstrated that transnasal pharyngoesophagogastroduodenoscopy (T-PEGD) is a safe and feasible procedure, and can be done without sedation. In this case report, T-PEGD was successfully performed in a patient with a fractured mandible immobilized with wires. We were successful in injecting epinephrine around a visible vessel

\section{K. S. Dua, E. Ruan, R. Shaker}

Dysphagia Institute and Division of Gastroenterology, Medical

College of Wisconsin, USA

Zablocki Veterans' Association Medical Center, Milwaukee, Wisconsin, USA

\section{References}

1. Leisser A, Delpre G, Kadish U. Through the nose with the gastroscope. Gastrointest Endosc 1990; 35: 77

2. Johnson DA, Cattau EL, Khan A, Newell DE, Chobanian SJ. Fiberoptic esophagogastroscopy via nasal intubation. Gastrointest Endosc 1987; 33:32-3.

3. Mitchell RG, Kerr RM, Ott DJ, Chen M. Transnasal endoscopic technique for feeding tube placement. Gastrointest Endosc 1992; 38: 596-7.

4. Shaker R. Unsedated trans-nasal pharyngoesophagogastroduodenoscopy (T-EGD): technique. Gastrointest Endosc 1994; 40: 346-8.

Corresponding Author

K. Dua, M. D.

GI Section/111 C

Zablocki VA Medical Center

5000 West National Avenue

Milwaukee, WI 53295

USA

Fax: +1-414-384-8480 